\title{
The correlation between tumor necrosis factor gene polymorphism and diabetic retinopathy.
}

\author{
Wang Jing1, Wang Xiuyan², Jiang Zhengxuan'1, Bao Ning ${ }^{1}$, Liu Heting ${ }^{1}$, Tao Liming ${ }^{\text {* }}$ \\ ${ }^{1}$ Department of Ophthalmology, the Second Affililatied Hospital of Anhui Medical University, Hefei, 230601, Anhui, \\ PR China \\ ${ }^{2}$ Department of Endocrinology, the Second Affililatied Hospital of Anhui Medical University, Hefei, 230601, Anhui, \\ PR China
}

\begin{abstract}
Aim: Some studies showed that tumor necrosis factor gene polymorphism related to the progression of diabetes mellitus. The aim of this study is to explore the correlation between the gene polymorphism of tumor necrosis factor and diabetic retinopathy in clinical cases.

Methods: We took the clinical cases as research subjects. The patients with type I and type II diabetes mellitus who had retinopathy and no retinopathy were 120 cases and 100 cases. At the same time, we analyzed gene polymorphism of tumor necrosis factor for all the patients (rs1800629).

Results: A allelic gene of $r \$ 1800629$ related to type I diabetes mellitus. We did not find significant correlation between $r s 1800629$ and diabetic retinopathy by adjusting age, gender, duration of diabetes mellitus, HbALc, high blood pressure, kidney disease and other variables.

Conclusion: This study has preliminarily confirmed that functional single nucleotide polymorphism $A$ in promoter region related to type I diabetes mellitus, which had no significant correlation with diabetic retinopathy.
\end{abstract}

Keywords: Tumor necrosis factor, Gene polymorphism, Diabetic retinopathy.

Accepted on November 15, 2017

\section{Introduction}

Diabetes mellitus (DM) is one of the most common diseases in clinic, which has high morbidity rate and mortality rate. Its main related complication is microangiopathy, including diabetic retinopathy (DR) and diabetic nephropathy (DN), which will cause a serious impact on the life quality of the patients [1]. Diabetic retinopathyis mainly due to that high blood glucose level promotes retinal capillary proliferation, which will lead to ablepsia if it is serious. Diabetic retinopathy is the local expression of systemic disease. The epidemiologic study showed that $80 \%$ patients with diabetes mellitus for more than 20 years would suffer from severe diabetic retinopathy $[2,3]$. In the new cases of blindness in the United States, more than $12 \%$ of them was induced by diabetes mellitus, and at the same time, it was also the main cause of blindness population aged 20-64 years of age. So its related research and treatment is always a hot spot in medical research [4-8]. The pathological changes of retinal capillary are mainly caused by a series of cascade reactions, which includes the formation of advanced glycation end products (AGEs) and persistent high blood sugar [9-11]. The interaction between AGEs and its receptors on the surface of macrophages and endothelial cells promotes the compound and secretion of multiple pro-inflammatory cytokines, including tumor necrosis factor (TNF- $\alpha)$. It was reported that TNF- $\alpha$ was mainly involved in the destruction of the blood retinal barrier and the formation of new blood vessels, so it was one of the main effective factors of diabetic retinopathy [12]. Many studies had reported that the polymorphism of tumor necrosis factor promoter related to some related diseases, and rs1800629 (TNF-308) was the most widely used single nucleotide polymorphism sites. Compared with the increased level of TNF in G allele cycle, TNF-308 A homozygous individuals with allele reduction, which was also bad for the prognosis of infectious diseases [13-16]. TNF gene was located near the 6 chromosome, which was human leucocyte antigen (HLA). In this study, we chose the clinical patients with type I and type II diabetes mellitus, and preliminarily explored the polymorphism of the gene promoter (rs 1800629) in the TNF regulatory region, compared and analyzed the correlation between its related effect and diabetic retinopathy, which could provide references and guidance for clinical treatment and basic research.

\section{Subjects and Methods}

This work was approved by the Ethic Committee of the Second Affililatied Hospital of Anhui Medical University. 


\section{Demographic information}

The clinical cases were research subjects, we chose 440 patients with diabetes mellitus who came to our hospital from January, 2015 to January, 2016. The number of patients with type I and type II diabetes mellitus were 220 cases respectively. In each group, there were 120 cases with retinopathy and 100 cases without diabetic retinopathy. The male female ratio of type I diabetes mellitus was 5:6, the average age was $58.6 \pm 15.2$ years old (47-69 years old), the average blood pressure was $138 / 81 \mathrm{mmHg}$ and the time of diabetes was $15.2 \pm 6.6$ years. The male female ratio of type II diabetes mellitus was $4: 7$, the average age was $61.2 \pm 16.6$ years old (45-71 years old), the average blood pressure was $137 / 82 \mathrm{mmHg}$ and the time of diabetes was $18.4 \pm 7.4$ years. All patients were from the same race. All the operations in this study were informed and agreed by patients, family members and the ethics committee of the Second Affililatied Hospital of Anhui Medical University.

\section{Choice criterion}

Firstly, selected the patients according to inclusion and exclusion criteria before the study, the inclusion criteria included: the age was over 18 years old; the patients who was receiving the treatment for diabetes mellitus (took antidiabetic agents or insulin orally); the patients with type II diabetes mellitus had been receiving hypoglycemic therapy for more than 5 years; the patients with combined heart, liver, lung, nervous system, hematological system and other serious illnesses or complications were excluded. The basic information of the patients was collected by the form of questionnaires. Evaluated the condition of the included retina according to the revised Early Treatment of Diabetic Retinopathy Standard (ETDRS). And sight-threatening diabetic retinopathy (STDR) was defined as at least one eye with non proliferative diabetic retinopathy, proliferative diabetic retinopathy and diabetic macular edema. While the control group defined it as the retinal grading never exceeds the minimum NPDR, no history of macular edema. The evaluation of eye ground was finished by a professional ophthalmologist alone, who knew nothing about the diabetes type information for patients.

\section{DNA analysis, data statistics and analysis}

Extracted DNA from whole blood, the specific operation was processed with QIAamp blood DNA kit (Qiagen company) according to product instructions. We used Sequenom mass spectrometer to type the gene of $r s 1800629$. SNP site was determined according to Hardy-Weinberg law of equilibrium. All the data and analysis were processed by SPSS 20.0 software (IBM SPSS Statistics 20.0, SPSS Inc., Armonk, NY). The continuous non parametric variables in the basic information of case group and control group were analyzed by Mann-Whitney U test. We chose $\chi^{2}$ test to analyze dichotomic variable. The correlation between SNP and DR was analyzed by Chi square test, while the multivariable analysis was analyzed by binary logistic regression. If $p<0.05$, then the differences had statistical significance.

\section{Results}

\section{Correlation among rs1800629, NPDR, PDR and DME}

We made multi factor Logistic regression analysis by controlling age, gender, duration of diabetes mellitus, glycosylated hemoglobin (HbAlc), hypertension and other variables, the result showed that there was no obvious correlation among rs 1800629, NPDR, PDR and DME for the patients with type I and type II diabetes mellitus. At the same time, in this study, there was no significant difference in the degree of fundus retinopathy for the patients with two kinds of diabetes mellitus (Figure 1).

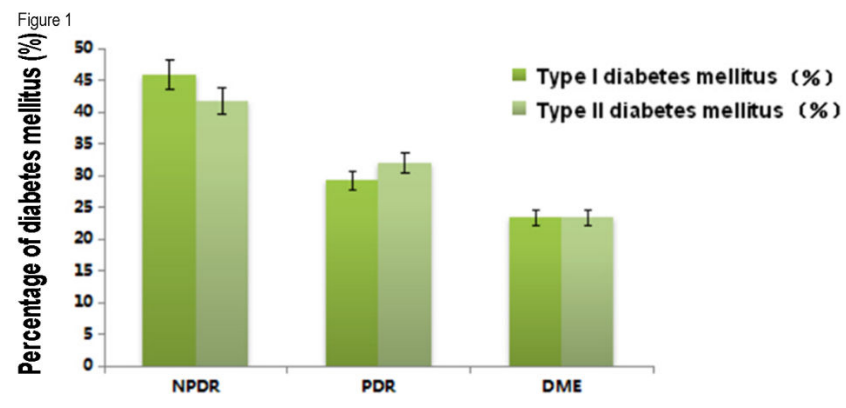

Figure 1. The comparison and analysis of different fundus retinopathy degrees for the patients with two kinds of diabetes mellitus. NPDR: Nonproliferative Diabetic Retinopathy; PDR: Proliferating Diabetic Retinopathy; DME: Diabetic Macular Edema.

\section{Rs1800629 was significantly related to the type of diabetes mellitus}

The result of this study showed that rs1800629 A allele frequency in the patients with type I diabetes mellitus increased obviously than that in the patients with type II diabetes mellitus (minimum allele frequency (MAF) of type I DM and type II DM was $23.6 \%$ and $16.1 \%$ respectively) [odds ratio $(\mathrm{OR})=0.62 ; 95 \%$ confidence interval $(\mathrm{CI})=0.53-0.71$; $\mathrm{p}<0.001]$, which was the same with previous research results. At the same time, we also found that the MAF of $r s 1800629$ increased significantly in the patients without high blood pressure $(\mathrm{OR}=0.848 ; 95 \% \mathrm{CI}=0.73-0.98 ; \mathrm{p}=0.025)$, young patients $(\mathrm{t}=-4.0 ; \mathrm{p}<0.001)$, the patients with long course of diabetes mellitus $(\mathrm{t}=2.44 ; \mathrm{p}=0.01)$. There differences indicated the characteristics of risk factors in the population with Type I diabetes mellitus. The above significance of differences were adjusted by Bonferroni ( $\mathrm{P}<0.025)$ (Table 1$)$. 
Table 1. The comparison and analysis of the correlation among rs1800629 (-308G>A), NPDR, PDR and DME for the patients with two kinds of diabetes mellitus.

\begin{tabular}{|c|c|c|c|c|c|c|c|c|}
\hline \multirow{3}{*}{$\begin{array}{l}\text { SNP } \\
r s 1800629\end{array}$} & \multicolumn{4}{|c|}{ Type I diabetes mellitus } & \multicolumn{4}{|c|}{ Type II diabetes mellitus } \\
\hline & Before adjustment & & After adjustment & & Before adjustment & & After adjustment & \\
\hline & OR $(95 \% \mathrm{Cl})$ & $P$ value & OR $(95 \% \mathrm{Cl})$ & $P$ value & OR $(95 \% \mathrm{Cl})$ & $P$ value & OR $(95 \% \mathrm{Cl})$ & $P$ value \\
\hline NPDR & $1.06(0.84-1.39)$ & 0.57 & $0.94(0.62-1.45)$ & 0.81 & $0.96(0.87-1.21)$ & 0.88 & $0.94(0.77-1.18)$ & 0.69 \\
\hline PDR & $1.15(0.86-1.61)$ & 0.26 & $1.05(0.61-1.61)$ & 0.93 & $0.92(0.72-1.09)$ & 0.56 & $0.93(0.71-1.29)$ & 0.56 \\
\hline DME & $0.96(0.68-1.38)$ & 0.96 & $1.37(0.80-2.28)$ & 0.21 & $0.96(0.71-1.19)$ & 0.55 & $0.95(0.77-1.19)$ & 0.68 \\
\hline
\end{tabular}

\section{Discussion}

Early detection of diabetic retinopathy has great significance for the children and adolescents with type I diabetes. At present, most researches were focused on the mechanism of cytokines in proliferative retinopathy, which indicated that high blood glucose level promoted the increasing expression of proinflammatory cytokine and it participated in the progression of retinopathy $[17,18]$. And TNF- $\alpha$ played a role of local cellular molecular signal aggregation in chronic inflammation of diabetic fundus.

In our study, We have found that $r s 1800629$ was related to type I diabetes mellitus. However, there was no significant correlation between $r s 1800629$ and diabetic retinopathy by adjusting age, gender, duration of diabetes mellitus, HbALc, high blood pressure, kidney disease and other variables. The previous study on the correlation between rs 1800629 promoter SNP and DR reported that there was no obvious correlation between the polymorphism of this gene and the risk of STDR in white race patient population [19], which was the same with the result of this study. While the latest study showed that rs 1800629 allel A related to the increased morbidity rate of PDR in Brazil population [16]. So it was not excluded the possibility of racial differences in the relationship. Functional studies indicated that TNF could be regarded as the biological marker of diabetic retinopathy. Compared with diabetic patients without retinopathy, type I diabetes mellitus combined with retinal pathology to increase TNF level in peripheral circulating serum [20,21]. At the same time, mRNA of tumor necrosis factor in vitreous body of patients and its expression of soluble receptors increased significantly. The animal research indicated that used angiogenin-1 could control the expression of tumor necrosis factor effectively, which was helpful to improve the prognosis of early diabetic retinopathy in rats [22-24].

More and more evidences showed the complex interreaction between the TNF in HLA III level area and other genes, the related studies might help to explain the genetic and functional differences [25]. Linkage disequilibrium of alleles in the major histocompatibility complex (MHC) region made low possibility of the correlation between SNPs and TNF phenotype in this region. The study assumed that possible alternative approaches of the TNF expression might exist, including the interaction among promoter methylation of TNF promoter in 3 'non translation region, -308 element and epigenetic control [26,27]. The further study on this kind of gene and expression regulation factor would be helpful to recognize the action mechanism of pro-inflammatory cytokine in diabetic retinopathy.

The recent research showed that the linkage disequilibrium occurred in tumor necrosis factor rs 1800629 allele and MHC haplotype HLA-A1-B8-DR3, which could explain functional correlation among allele A, high $T N F$ expression and insulindependent diabetes. upto now, there was no specific factor that could indicated tumor necrosis factor SNPs could increase the susceptibility of type I diabetes mellitus [28,29].

\section{Conclusion}

In conclusion, $r s 1800629$ (TNF-308A) allelic gene was related to type I diabetes mellitus, we found no correlation between gene polymorphism in promoter region and two kinds of diabetes mellitus. At present, the study showed that the systemic or local increased TNF factor level expression related to diabetic retinopathy. But further studies are needed to explore the complex interactions among TNF gene, HLA haplotype and epigenetic modification to clarify the specific impact of it on diabetic microangiopathy. Our findings provide suggestive evidence that polymorphisms in the $T N F$ gene may be potentially involved in the pathogenesis of diabetic retinopathy and should be investigated further.

\section{References}

1. Madonna R, Balistreri CR, Geng YJ, De Caterina R. Diabetic microangiopathy: Pathogenetic insights and novel therapeutic approaches. Vascul Pharmacol 2017; 90: 1-7.

2. Gustavsson C, Agardh E, Bengtsson B, Agardh CD. TNFalpha is an independent serum marker for proliferative retinopathy in type 1 diabetic patients. $\mathrm{J}$ Diabetes Complicat 2008; 22: 309-316.

3. Limb GA, Hollifield RD, Webster L, Charteris DG, Chignell AH. Soluble TNF receptors in vitreoretinal proliferative disease. Invest Ophth Vis Sci 2001; 42: 1586-1591.

4. Yang N, Zhang W, He T, Xing Y. Suppression of retinal neovascularization by inhibition of galectin-1 in a murine model of oxygen-induced retinopathy. J Ophthalmol 2017; 2017: 5053035 .

5. Hirahara S, Nozaki M, Ohbayashi M, Hasegawa N, Ozone D, Ogura Y. Suppression of retinal neovascularization by anti-CCR3 treatment in an oxygen-induced retinopathy model in mice. Ophthalmic Res 2017; 58: 56-66. 
6. Campochiaro PA, Peters KG. Targeting Tie2 for treatment of diabetic retinopathy and diabetic macular edema. Curr Diab Rep 2016; 16: 126.

7. Xing Q, Zhang G, Kang L, Wu J, Chen H, Liu G, Zhu R, Guan H, Lu P. The suppression of kallistatin on highglucose-induced proliferation of retinal endothelial cells in diabetic retinopathy. Ophthalmic Res 2017; 57: 141-149.

8. Tikhonenko M, Lydic TA, Opreanu M, Li Calzi S, Bozack S, McSorley KM, Sochacki AL, Faber MS, Hazra S, Duclos S, Guberski D, Reid GE, Grant MB, Busik JV. N-3 polyunsaturated Fatty acids prevent diabetic retinopathy by inhibition of retinal vascular damage and enhanced endothelial progenitor cell reparative function. PLoS One 2013; 8: e55177.

9. Chen YH, Chou HC, Lin ST, Chen YW, Lo YW, Chan HL. Effect of high glucose on secreted proteome in cultured retinal pigmented epithelium cells: its possible relevance to clinical diabetic retinopathy. J Proteomics 2012; 77: 111-128.

10. Milne R, Brownstein S. Advanced glycation end products and diabetic retinopathy. Amino Acids 2013; 44: 1397-1407.

11. Feng Y, Gross S, Chatterjee A, Wang Y, Lin J, Hammes HP. Transcription of inflammatory cytokine $\mathrm{TNF} \alpha$ is upregulated in retinal angiogenesis under hyperoxia. Cell Physiol Biochem 2016; 39: 573-583.

12. Kaidonis G, Abhary S, Daniell M, Gillies M, Fogarty R, Petrovsky N, Jenkins A, Essex R, Chang JH, Pal B, Hewitt AW, Burdon KP, Craig JE. Genetic study of diabetic retinopathy: recruitment methodology and analysis of baseline characteristics. Clin Experiment Ophthalmol 2014; 42: 486-493.

13. Lindholm E, Bakhtadze E, Cilio C, Agardh E, Groop L, Agardh CD. Association between LTA, TNF and AGER polymorphisms and late diabetic complications. PLoS ONE 2008; 3: e2546.

14. Wang N, Huang K, Zou H, Shi Y, Zhu J, Tang W, Xu X. No association found between the promoter variants of TNF- $\alpha$ and diabetic retinopathy in Chinese patients with type 2 diabetes. Curr Eye Res 2008; 33: 377-383.

15. Meng N, Zhang Y, Li H, Ma J, Qu Y. Association of tumor necrosis factor alpha promoter polymorphism (TNF- $\alpha 238$ G/A and TNF- $\alpha 308 \mathrm{G} / \mathrm{A}$ ) with diabetic mellitus, diabetic retinopathy and diabetic nephropathy: a meta-analysis. Curr Eye Res 2014; 39: 194-203.

16. Sesti LFC, Crispim D, Canani LH, Polina ER, Rheinheimer J, Carvalho PS, Gross JL, Santos KG. The-308G>a polymorphism of the TNF gene is associated with proliferative diabetic retinopathy in Caucasian Brazilians with type 2 diabetes. Invest Ophth Vis Sci 2015; 56: 1184-1190.

17. Demirbas B, Guler S, Cakir B, Culha C, Aral Y. Plasma tumor necrosis factor-alpha levels and insulin resistance in nondiabetic hypertensive subjects. Horm Res 2002; 58: 283-286.
18. Mishima Y, Kuyama A, Tada A, Takahashi K, Ishioka T, Kibata M. Relationship between tumor necrosis factoralpha and insulin resistance in obese men with type 2 diabetes mellitus. Diabetes Res Clin Pract 2001; 52: 119-123.

19. Gwozdziewiczova S, Lichnovska R, Ben Yahia R, Chlup R, Hrebicek J. TNF $\alpha$ in the development of insulin resistance and other disorders in metabolic syndrome. Biomed Pap Med Fac Uni Palacky Olmouc Czech Repub 2005; 149: 109-119.

20. Yudkin JS, Panahloo A, Stehouwer C, Emeis JJ, Bulmer K, Mohamed-Ali V, Denver AE. The influence of improved glycaemic control with insulin and sulphonylureas on acute phase and endothelial markers in type II diabetic subjects. Diabetologia 2000; 43: 1099-1106.

21. Moller DE. Potential role of TNF alpha in the pathogenesis of insulin resistance and type 2 diabetes. Trends Endocrinol Metab 2000; 11: 212-217.

22. Kang MK, Park SH, Kim YH, Lee EJ, Antika LD, Kim DY, Choi YJ, Kang YH. Dietary Compound Chrysin Inhibits Retinal Neovascularization with Abnormal Capillaries in $\mathrm{db} / \mathrm{db}$ Mice. Nutrients 2016; 8: E782.

23. Zou C, Shao J. Role of adipocytokines in obesityassociated insulin resistance. J Nutr Biochem 2008; 19: 277-286.

24. Pepys MB, Hirschfield GM, Tennent GA, Gallimore JR, Kahan MC, Bellotti V, Hawkins PN, Myers RM, Smith MD, Polara A, Cobb AJ, Ley SV, Aquilina JA, Robinson CV, Sharif I, Gray GA, Sabin CA, Jenvey MC, Kolstoe SE, Thompson D, Wood SP. Targeting C- reactive protein for the treatment of cardiovascular disease. Nature 2006; 440: 1217-1221.

25. Schram MT, Chaturvedi N, Schalkwijk C, Giorgino F, Ebeling P, Fuller JH, Stehouwer CD; EURODIAB Prospective Complications Study. Vascular risk factors and markers of endothelial function as determinants of inflammatory markers in type 1 diabetes: the EURODIAB Prospective Complications Study. Diabetes Care 2003; 26: 2165-2173.

26. Giorgino F, Laviola L, Cavallo Perin P, Solnica B, Fuller J, Chaturvedi N. Factors associated with progression to macroalbuminuria in microalbuminuric type 1 diabetic patients: the EURODIAB Prospective Complications Study. Diabetologia 2004; 47: 1020-1028.

27. Sugimoto H, Shikata K, Wada J, Horiuchi S, Makino H. Advanced glycation end products-cytokine-nitric oxide sequence pathway in the development of diabetic nephropathy: aminoguanidine ameliorates the overexpression of tumour necrosis factor- $\alpha$ and inducible nitric oxide synthase in diabetic rat glomeruli. Diabetologia 1999; 42: 878-886.

28. Doganay S, Evereklioglu C, Er H, Türköz Y, Sevinç A, Mehmet N, Savli H. Comparison of serum NO, TNF- $\alpha$, IL-1 $\beta$, sIL-2R, IL-6 and IL-8 levels with grades of retinopathy in patients with diabetes mellitus. Eye 2002; 16: $163-170$. 
29. Wan TT, Li XF, Sun YM, Li YB, Su Y. Recent advances in understanding the biochemical and molecular mechanism of diabetic retinopathy. Biomed Pharmacother 2015; 74: 145-147.

\section{*Correspondence to}

Tao Liming

Department of Ophthalmology

The Second Affililatied Hospital of Anhui Medical University

PR China 